\title{
The Association between Mental Health Disorders and History of Unintended Pregnancy among Women Veterans
}

\author{
Colleen P. Judge-Golden, $B A^{7}$, Sonya Borrero, MD, $M S^{2,3}$, Xinhua Zhao, PhD'², Maria K. Mor, PhD ${ }^{2,4}$, \\ and Lisa S. Callegari, MD, MPH,
}

'University of Pittsburgh School of Medicine, Pittsburgh, PA, USA; ${ }^{2}$ Center for Health Equity, Research, and Promotion, VA Pittsburgh Health Care System, Pittsburgh, PA, USA; ${ }^{3}$ Center for Research on Health Care, University of Pittsburgh, Pittsburgh, PA, USA; ${ }^{4}$ Department of Biostatistics, Graduate School of Public Health, University of Pittsburgh, Pittsburgh, PA, USA; ${ }^{5}$ Department of Obstetrics \& Gynecology, University of Washington School of Medicine, Seattle, WA, USA; ${ }^{6}$ Health Services Research and Development, VA Puget Sound Health Care System, Seattle, WA, USA.

\begin{abstract}
BACKGROUND: Women Veterans have high rates of mental health disorders and other psychosocial factors which may render this population particularly vulnerable to negative health outcomes associated with unintended pregnancy.
\end{abstract}

OBJECTIVE: The objective of our study was to assess the relationship between self-reported mental illness and history of unintended pregnancy among women Veterans.

DESIGN AND PARTICIPANTS: Data are from a nationally representative, cross-sectional telephone survey of women Veterans, ages 18-45, who used VA for primary care within 12 months prior to interview (survey completion rate $83 \%$ ).

MAIN MEASURES: Predictors were self-report of any and number of mental health disorders (depression, anxiety, post-traumatic stress disorder, bipolar disorder, or schizophrenia). Outcomes were any and number of unintended pregnancies. Multivariable logistic and negative binomial regression were used to assess relationships between mental illness and unintended pregnancy. To assess women's current risk of unintended pregnancy, we examined associations between any mental health disorder and contraceptive use at last sex among heterosexually active women not desiring pregnancy.

KEY RESULTS: Among 2297 women Veterans, 1580 $(68.8 \%)$ reported a history of at least one mental health disorder, with $20.1,21.6$, and $27.0 \%$ reporting one, two, or three or more conditions, respectively. Any history of unintended pregnancy was reported by 1315 women (57.3\%); $28.3 \%$ reported one, $15.6 \%$ reported two, and $13.4 \%$ reported three or more. Compared to women with no mental illness, women with any mental health disorder were more likely to report any unintended pregnancy (60.3 vs. 50.5\%; adjusted OR 1.40; 95\% CI 1.15, 1.71) and to have experienced greater numbers of unintended pregnancies (adjusted incidence rate ratio 1.29; 95\% CI $1.15,1.44)$. Increasing numbers of mental health disorders were associated with greater numbers of unintended

Electronic supplementary material The online version of this article (https://doi.org/10.1007/s11606-018-4647-8) contains supplementary material, which is available to authorized users.

Received March 26, 2018

Revised July 18, 2018

Accepted August 21, 2018

Published online September 5, 2018 pregnancies. Contraceptive use and method efficacy at last sex did not differ by mental health status.

CONCLUSIONS: Women Veterans with mental health disorders are more likely to have experienced any and greater numbers of unintended pregnancies than Veterans without mental health disorders.

KEY WORDS: mental health; women Veterans; unintended pregnancy.

$\mathrm{J}$ Gen Intern Med 33(12):2092-9

DOI: $10.1007 / \mathrm{s} 11606-018-4647-8$

(c) Society of General Internal Medicine 2018

\section{INTRODUCTION}

Women Veterans of reproductive age comprise the fastest growing population within the Veterans Affairs (VA) Healthcare System. ${ }^{1,2}$ Despite availability of the full range of contraceptive methods through VA, over one third of pregnancies among female VA-users are unintended, comparable to age-adjusted national statistics. ${ }^{3}$ Unintended pregnancy is associated with adverse health behaviors and outcomes such as substance use during pregnancy, preterm birth, and low birth weight. ${ }^{4}$ Compared to women in the general population, women Veterans have high rates of both chronic medical conditions and mental health disorders, including depression, anxiety, post-traumatic stress disorder (PTSD), and substance use disorders. ${ }^{1,5,6}$ This high prevalence of medical and psychiatric comorbidities, along with high rates of adverse psychosocial factors including homelessness and history of sexual trauma, ${ }^{1,6-8}$ may place this population at particularly high risk for the negative outcomes associated with unintended pregnancy. However, little is known about factors associated with unintended pregnancy among women Veterans.

Mental illness during pregnancy is associated with increased risk of pregnancy-related morbidity including preterm birth and low birth weight, ${ }^{9-11}$ which may compound negative outcomes associated with unintended pregnancy. However, few studies directly assess the relationship between mental health disorders and experience of unintended pregnancy, ${ }^{12,13}$ and none in Veterans. Improved understanding of the relationship between mental illness and unintended pregnancy among 
women Veterans is essential to inform efforts to improve reproductive health in this vulnerable population. We therefore evaluated associations between self-reported mental health disorders and history of unintended pregnancy, using data from a nationally representative survey of women Veterans.

\section{MATERIALS AND METHODS}

\section{Study Design and Population}

Data are from the Examining Contraceptive Use and Unmet Need among Women Veterans (ECUUN) study; complete methodology is reported elsewhere. ${ }^{3}$ Briefly, ECUUN included a cross-sectional, telephone-based survey with a random national sample of 2302 women Veterans ages 18-45 who used VA for primary care within 12 months prior to study interview. VA administrative data were used to identify a random sample of potential participants quarterly, and selected individuals were mailed study invitation packets. Women who did not opt out of study participation were contacted by telephone to determine interest in participating, assess eligibility criteria, and conduct informed consent procedures. The institutional review boards of VA Pittsburgh Healthcare System and the University of Pittsburgh approved this study.

Participants completed computer-assisted telephone interviews between April 2014 and January 2016, and the survey completion rate was $83 \%$ among enrolled participants. Study participants were similar to non-participants from the sampling frame in terms of age, race/ethnicity, marital status, income, geographic region, and presence of medical and mental illness, suggesting that the ECUUN sample is representative of the larger population of reproductive-aged female VAusers. $^{3}$ This analysis excludes five participants who provided no information about current or prior pregnancies, for a sample size of 2297 women.

\section{Measures}

History of mental health disorders was assessed via self-report on the survey instrument. Participants were asked, "which, if any, of the following have you been diagnosed with or received treatment for in your lifetime?" and were asked to select all that apply, or none, of the following conditions: major depression/clinical depression, bipolar disorder, posttraumatic stress disorder, schizophrenia, and anxiety/panic disorder.

We assessed pregnancy intention (unwanted, occurring sooner than desired, occurring later than desired, occurring at the right time, or “don't know/didn't care") using two standard questions for each pregnancy, including current pregnancies. Per convention, we considered as unintended those pregnancies described as unwanted (i.e., when no future pregnancies were desired) or occurring sooner than desired. ${ }^{14}$ Complete pregnancy data, including intention status and outcomes for completed pregnancies (live birth, miscarriage/stillbirth, abortion, or ectopic pregnancy/other), was recorded for the first ten pregnancies reported $(n=6$ participants reported $>10$ pregnancies). Due to an error in skip patterns, intention status of a current pregnancy was not assessed for 5 of 10 currently pregnant women who also endorsed a history of diagnosed infertility; information about past pregnancies, but not the current pregnancy, are included in analyses for these five women.

Participants reported all contraceptive methods used at last vaginal intercourse, and we categorized responses by level of contraceptive effectiveness: highly effective methods (male and female sterilization, intrauterine device (IUD), contraceptive implant), moderately effective methods (pill, patch, ring, Depo-Provera), least effective methods (condom, diaphragm, cervical cap, sponge, spermicide, withdrawal, and fertility awareness methods), or no method. Women reporting use of more than one method were classified according to their most effective method.

We examined the following covariates using survey data: age, race/ethnicity, marital status, education, religion, annual household income, additional (non-VA) insurance, deployment history, and history of military sexual trauma (MST). Geographic region was determined using VA administrative data.

\section{Statistical Analysis}

Our primary independent variables were any mental health disorder and number of mental health disorders $(0,1,2$, or 3 or more). Outcomes were any history of unintended pregnancy and number of unintended pregnancies (0 to 10). We generated descriptive statistics to describe sociodemographic characteristics of the total study population and by presence of any mental health disorder, and a pie chart to depict the detailed distribution of mental health disorders, including individual disorders among women reporting one condition, and combinations of conditions among women reporting a history of more than one mental health disorder.

We summarized the frequencies and proportions of women reporting any history of unintended pregnancy and number of unintended pregnancies; this variable was collapsed to $0,1,2$, or 3 or more for descriptive purposes due to data skewness. We used chi-square tests to test differences in any unintended pregnancy across any mental health disorder, and the Jonchkheere-Terpstra test for ordered alternatives to test trends between number of mental health disorders and number of unintended pregnancies. We used multivariable logistic regression to assess the adjusted relationships between the two predictors (any mental health disorder and number of mental health disorders) and any history of unintended pregnancy. Multivariable negative binomial regression was used to assess relationships between mental health disorders and number of unintended pregnancies. We selected negative binomial regression because Poisson regression models displayed significant over-dispersion, although incidence rate ratio point estimates were unchanged. We included all assessed covariates in regression models based on a priori theoretical or empirical 
associations with mental illness or unintended pregnancy. Adjusted odds ratios (aOR) and 95\% confidence intervals (CI) are reported.

We performed exploratory bivariate analyses using chisquare tests to examine relationships between any unintended pregnancy and individual mental health disorders as well as the most frequent combinations of co-reported disorders. Due to the high prevalence of women endorsing more than one mental health disorder and small sample sizes of individual conditions, we were unable to assess adjusted associations of specific mental health disorders or combinations with unintended pregnancy. We also assessed the association between any mental health disorder and the outcome of individual unintended pregnancies, using multinomial logistic regression models with random effects to account for clustering of pregnancies by woman.

In a secondary analysis to assess women's ongoing risk of unintended pregnancy, we examined the relationship between any mental health disorder and contraceptive use and method effectiveness at last intercourse. This analysis included a subsample of participants who were heterosexually active within 1 year prior to interview, not currently pregnant or attempting to conceive, and with no history of diagnosed infertility or hysterectomy $(n=1339)$. Chi-square tests were used to assess bivariate relationships. We used multivariable logistic and multinomial logistic regression to assess the adjusted associations of any mental health disorder with any contraceptive use and contraceptive efficacy, respectively. All assessed covariates were included in models based on a priori theoretical or empirical associations with mental illness or contraceptive use. All analyses were conducted in Stata 14.

\section{RESULTS}

In our sample of 2297 women Veterans, the mean age was 34.7 years (range $21-45$ ); $51.6 \%$ of women were nonHispanic white, $28.8 \%$ non-Hispanic black, $12.4 \%$ Hispanic, and $7.1 \%$ non-Hispanic other, including multiracial, Asian, Pacific Islander, and Native American (Table 1). Overall, 1580 women $(68.8 \%)$ reported a history of at least one mental health disorder; 462 women $(20.1 \%)$ reported a single condition, $497(21.6 \%)$ reported two, and $621(27.0 \%)$ reported three or more conditions. Anxiety was the most commonly reported condition $(n=1145,49.9 \%)$, followed by depression $(n=1111,48.4 \%)$ and PTSD $(n=951,41.4 \%)$. The distribution of single and co-reported mental health disorders are shown in Fig. 1. Compared to women with no mental illness, women with at least one mental health disorder were more likely to be non-Hispanic white; divorced, separated or widowed; and to report a history of MST.

A total of 1315 women $(57.3 \%)$ reported ever experiencing an unintended pregnancy; $28.3 \%$ reported one, $15.6 \%$ reported two, and $13.4 \%$ reported three or more (Table 2). Compared to women with no mental illness, women with any mental health disorder were more likely to have experienced any unintended pregnancy $(60.3$ vs. $50.5 \%, p<0.001)$ and reported greater numbers of unintended pregnancies (p-fortrend $<0.001$ ). Increasing number of mental health disorders was significantly associated with increasing number of unintended pregnancies ( $\mathrm{p}$-for-trend $<0.001$ ).

In adjusted analyses (Table 3), women with any mental health disorder had increased odds of having experienced any unintended pregnancy compared to women with no mental health disorders (aOR 1.40; 95\% CI 1.15, 1.71). Any mental health disorder was also associated with increased number of unintended pregnancies compared to women with no mental illness (adjusted Incidence Rate Ratio (aIRR) 1.29; $95 \%$ CI 1.15, 1.44). Greater numbers of mental health disorders were associated with greater numbers of unintended pregnancies compared to women with no mental health disorders.

The bivariate associations of any unintended pregnancy with individual and co-reported mental health disorders are shown in the Appendix. Depression alone; depression with anxiety; depression with anxiety and PTSD; and depression with anxiety, PTSD, and bipolar disorder were all associated with any history of unintended pregnancy compared to no mental health disorder. All other individual conditions and combinations trended toward higher frequencies of unintended pregnancy compared to no mental illness, but comparisons were non-significant.

Of 2489 completed unintended pregnancies reported by 1308 women, 1437 resulted in a live birth $(57.7 \%), 502$ (20.2\%) ended in abortion, 494 (19.9\%) resulted in miscarriage or stillbirth, and $56(2.3 \%)$ were ectopic or had other outcomes. Pregnancy outcomes were not significantly different according to mental health status, while adjusting for clustering of pregnancies by woman $(p=0.07)$ (Fig. 2).

Our secondary analysis of contraceptive use at last sex included 1339 heterosexually active women not desiring pregnancy (Table 4). There were no statistically significant differences in any contraceptive use at last sex among women with any mental health disorder versus none (93.8 vs. $95.6 \%$, respectively, $p=0.17$ ), or in method efficacy by mental health status among the 1264 women who used any contraception $(p=0.08)$. In adjusted analyses, there were no differences in any versus no contraceptive use (aOR $0.74 ; 95 \%$ CI 0.42 , 1.29 ), or in method efficacy among contraceptive users.

\section{DISCUSSION}

In this nationally representative sample of female VA-users of reproductive age, over two thirds of women Veterans reported a history of at least one mental health disorder, and these women were more likely to report ever experiencing an unintended pregnancy compared to women without mental illness. Women with mental health disorders had also experienced higher numbers of unintended pregnancies compared to 
Table 1 Demographic characteristics of total sample and by presence of $\geq 1$ mental health disorder

\begin{tabular}{|c|c|c|c|}
\hline \multirow[t]{2}{*}{ Characteristic } & \multirow{2}{*}{$\begin{array}{l}\text { Total } \\
n=2297\end{array}$} & \multicolumn{2}{|c|}{$\geq 1$ Mental health disorder } \\
\hline & & $\begin{array}{l}\text { No } \\
n=717 \\
(31.2 \%)\end{array}$ & $\begin{array}{l}\text { Yes } \\
n=1580 \\
(68.8 \%)\end{array}$ \\
\hline \multicolumn{4}{|l|}{ Age } \\
\hline $20-29$ & $452(19.7)$ & $157(21.9)$ & $295(18.7)$ \\
\hline $30-34$ & $686(29.9)$ & 207 (28.9) & $479(30.3)$ \\
\hline $35-39$ & $584(25.4)$ & $176(24.5)$ & $408(25.8)$ \\
\hline $40-45$ & $575(25.0)$ & $177(24.7)$ & $398(25.2)$ \\
\hline \multicolumn{4}{|l|}{ Race* $^{*}$} \\
\hline Non-Hispanic White & 1186 (51.6) & $325(45.3)$ & $861(54.5)$ \\
\hline Non-Hispanic Black & $662(28.8)$ & $236(32.9)$ & $426(27.0)$ \\
\hline Hispanic & $285(12.4)$ & $97(13.5)$ & 188 (11.9) \\
\hline Non-Hispanic Other & $164(7.1)$ & $59(8.2)$ & $105(6.7)$ \\
\hline \multicolumn{4}{|l|}{ Marital status* } \\
\hline Single, never married & $534(23.3)$ & $209(29.2)$ & $325(20.6)$ \\
\hline Married or cohabitating & $1149(50.1)$ & $368(51.3)$ & $781(49.5)$ \\
\hline Divorced/separated/widowed & $612(26.7)$ & $140(19.5)$ & $472(29.9)$ \\
\hline \multicolumn{4}{|l|}{ Education } \\
\hline College degree or higher & $1218(53.0)$ & $395(55.1)$ & $823(52.1)$ \\
\hline \multicolumn{4}{|l|}{ Religion* } \\
\hline No religion & $393(17.2)$ & $121(16.9)$ & $272(17.3)$ \\
\hline Protestant & $313(13.7)$ & $101(14.1)$ & $212(13.5)$ \\
\hline Catholic & $269(11.7)$ & $100(14.0)$ & $169(10.7)$ \\
\hline Other Christian & $1001(43.7)$ & $328(45.8)$ & $673(42.7)$ \\
\hline Other religion & $316(13.8)$ & $66(9.2)$ & $250(15.9)$ \\
\hline \multicolumn{4}{|l|}{ Annual household income } \\
\hline$\$ 0$ to $\$ 19,999$ & $460(20.3)$ & $130(18.4)$ & $330(21.1)$ \\
\hline$\$ 20,000$ to $\$ 59,999$ & $1229(54.1)$ & $373(52.8)$ & $856(54.7)$ \\
\hline$\geq \$ 60,000$ & $583(25.7)$ & $203(28.8)$ & $380(24.3)$ \\
\hline \multicolumn{4}{|l|}{ Census region } \\
\hline Northeast & $200(8.7)$ & $57(8.0)$ & $143(9.1)$ \\
\hline Midwest & $408(17.8)$ & $116(16.2)$ & $292(18.5)$ \\
\hline South & $1220(53.1)$ & $393(54.8)$ & $827(52.3)$ \\
\hline West & $469(20.4)$ & $151(21.1)$ & $318(20.1)$ \\
\hline Has dual (non-VA) insurance & $1196(52.1)$ & $364(50.8)$ & $832(52.7)$ \\
\hline Ever deployed & $1272(55.5)$ & $403(56.3)$ & $869(55.1)$ \\
\hline History of military sexual trauma* & $1262(54.9)$ & $246(34.3)$ & $1016(64.3)$ \\
\hline \multicolumn{4}{|l|}{ Number of mental health disorders } \\
\hline 0 & $717(31.2)$ & $717(100)$ & - \\
\hline 1 & $462(20.1)$ & - & $462(29.2)$ \\
\hline 2 & 497 (21.6) & - & $497(31.5)$ \\
\hline 3 or more & $621(27.0)$ & - & $621(39.3)$ \\
\hline \multicolumn{4}{|l|}{ Type of mental health disordert } \\
\hline Depression & $1111(48.4)$ & - & $1111(70.3)$ \\
\hline Anxiety & $1145(49.9)$ & - & $1145(72.5)$ \\
\hline PTSD & 951 (41.4) & - & $951(60.2)$ \\
\hline Bipolar disorder & $208(9.1)$ & - & $208(13.2)$ \\
\hline Schizophrenia & $25(1.1)$ & & $25(1.6)$ \\
\hline
\end{tabular}

Data are $n(\%)$. Missing data (unknown/refused to answer): marital status $(n=2)$, religion $(n=5)$, income $(n=25)$, insurance $(n=1)$, ever deployed $(n=4) . p$ values are from chi-square tests

$* p<0.001$

†Includes women reporting multiple conditions

women without mental illness, and increasing numbers of mental health disorders were associated with increasing numbers of unintended pregnancies. Specific outcomes of unintended pregnancies were similar among women with and without mental health diagnoses. Our results suggest that women Veterans with mental health disorders may be at increased risk of unintended pregnancy, although underlying mechanisms remain unclear.

Prior research in non-Veteran populations supports our finding of an association between mental illness and unintended pregnancy. A few prospective studies have found positive associations between symptoms of mental illness, particularly depression, and subsequent unintended pregnancy. ${ }^{12,13}$ That mental health disorders and particularly active symptoms have been identified as predictors of contraceptive nonuse, reduced adherence, and method discontinuation ${ }^{15-19}$ suggests a potential mechanism for this observation. Furthermore, women with mental health disorders may be more likely to express ambivalent attitudes toward planning or avoiding pregnancy, ${ }^{20,21}$ which are also associated with reduced or inconsistent use of contraception. $^{22,23}$ Importantly, while mental health symptoms may contribute to a woman's prospective risk of unwanted or mistimed pregnancy, unintended pregnancy and resultant childbearing has also been found to be predictive of incident or worsening mental illness in the pre- or perinatal periods, ${ }^{24-26}$ as well as later in life. ${ }^{27}$ Thus, the directionality of a potential 


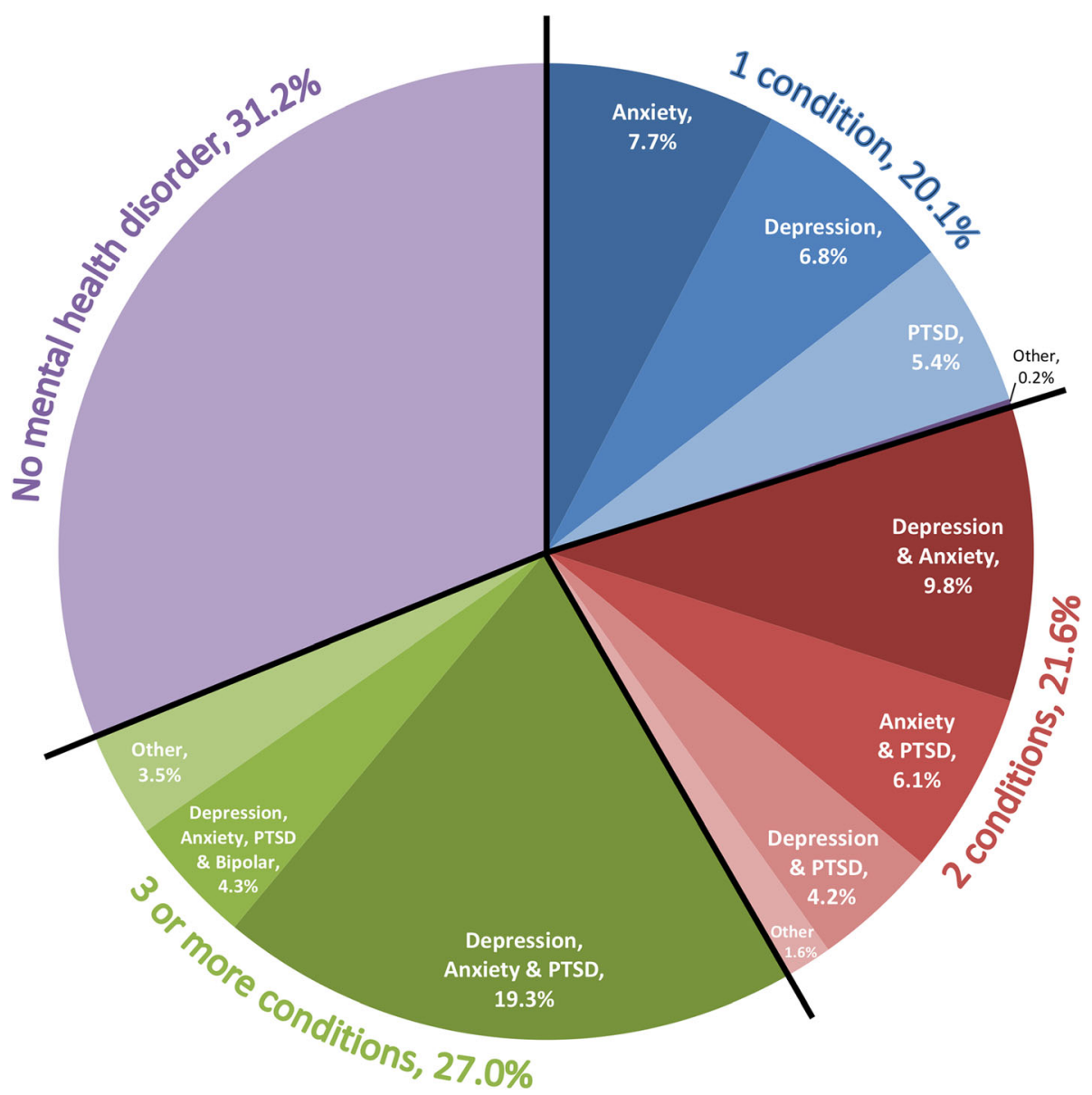

Fig. 1 Number and types of mental health disorders among 2297 women Veterans who use VA for primary care

causal relationship between mental illness and unintended pregnancy remains obscured. Due to the retrospective and cross-sectional nature of our study, we are also unable to draw conclusions regarding the temporal relationship between mental health symptoms or diagnoses and unintended pregnancy.

Similar to women in the general population, female VAusers with mental health disorders have also been found to have reduced rates of adherence to user-dependent contraceptive methods, ${ }^{16,28}$ which could potentially account for the higher rates of unintended pregnancy observed in our study.
However, other research suggests that women Veterans with mental health disorders are more likely than Veterans with no mental illness to use highly effective, long-acting contraceptive methods such as intrauterine devices or contraceptive implants, which do not rely on patient adherence. ${ }^{29}$ In our study, we found that contraceptive method use and efficacy at last sex was similar among women with and without mental health disorders. Rather than exposing a disparity, our findings indicate a degree of equity in current contraceptive provision by VA irrespective of mental health status, and suggest similar

Table 2 Number of unintended pregnancies by number of mental health disorders

\begin{tabular}{|c|c|c|c|c|c|}
\hline & \multirow{2}{*}{$\begin{array}{l}\text { Any unintended } \\
\text { pregnancy } n(\%)\end{array}$} & \multicolumn{4}{|c|}{ Number of unintended pregnancies } \\
\hline & & $\begin{array}{l}0 \\
n(\%)\end{array}$ & $\begin{array}{l}1 \\
n(\%)\end{array}$ & $\begin{array}{l}2 \\
n(\%)\end{array}$ & $\begin{array}{l}3+ \\
n(\%)\end{array}$ \\
\hline Total & $1315(57.2)$ & $982(42.8)$ & $649(28.3)$ & $359(15.6)$ & $307(13.4)$ \\
\hline \multicolumn{6}{|l|}{ Number of mental health disorders* } \\
\hline 0 mental health disorders $(n=717,31.2 \%)$ & $362(50.5)$ & $355(49.5)$ & $198(27.6)$ & $97(13.5)$ & $67(9.3)$ \\
\hline 1 mental health disorder $(n=462,20.1 \%)$ & $272(58.9)$ & $190(41.1)$ & $141(30.5)$ & $75(16.2)$ & $56(12.1)$ \\
\hline 2 mental health disorders $(n=497,21.6 \%)$ & $296(59.6)$ & $201(40.4)$ & $142(28.6)$ & $82(16.5)$ & $72(14.5)$ \\
\hline 3 or more mental health disorders $(n=621,27.0 \%)$ & $385(62.0)$ & $236(38.0)$ & $168(27.1)$ & $105(16.9)$ & $112(18.0)$ \\
\hline Any mental health disorder $(n=1580,68.8 \%)$ & $953(60.3)$ & $627(39.7)$ & $451(28.5)$ & $262(16.6)$ & $240(15.2)$ \\
\hline
\end{tabular}

*p-for-trend $<0.001$ for any unintended pregnancy by number of mental health disorders $(0,1,2$, or $\geq 3$ ). p-for-trend $<0.001$ for number of unintended pregnancies $(0,1,2$, or $\geq 3$ ) by number of mental health disorders $(0,1,2$, or $\geq 3$ )

$t p<0.001$ for any unintended pregnancy by any vs. no mental health disorders; $p$ value from chi-square test. p-for-trend $<0.001$ for number of unintended pregnancies $(0,1,2$, or $\geq 3)$ by any vs. no mental health disorders 
Table 3 Adjusted associations between mental health disorders and history of unintended pregnancy

\begin{tabular}{|c|c|c|}
\hline & Any unintended pregnancy* & Number of unintended pregnancies $\dagger$ \\
\hline & $\begin{array}{l}\text { Adjusted OR } \\
(95 \% \text { CI })\end{array}$ & $\begin{array}{l}\text { Adjusted IRR } \\
\text { (95\% CI) }\end{array}$ \\
\hline \multicolumn{3}{|l|}{ Model 1: number of mental health disorders } \\
\hline 0 mental health disorders & REF & REF \\
\hline 1 mental health disorder & $1.38(1.07,1.78)$ & $1.18(1.02,1.36)$ \\
\hline 2 mental health disorders & $1.37(1.07,1.77)$ & $1.29(1.12,1.48)$ \\
\hline$\geq 3$ mental health disorders & $1.44(1.12,1.84)$ & $1.39(1.22,1.59)$ \\
\hline Model 2: any mental health disorder vs. none & $1.40(1.15,1.71)$ & $1.29(1.15,1.44)$ \\
\hline
\end{tabular}

OR odds ratio, IRR incidence rate ratio

*Logistic regression models of any unintended pregnancy vs. none by number (0, 1, 2, or $\geq 3$ ) or any mental health disorders vs. no mental health disorders

$\dagger$ Negative binomial regression models of number of unintended pregnancies $(0,1,2,3, \ldots, 10)$ by number $(0,1,2$, or $\geq 3)$ or any mental health disorders vs. no mental health disorders

$\ddagger$ Adjusted for age, racelethnicity, marital status, education, annual household income, religion, additional (non-VA) insurance, census region, deployment history, and history of military sexual trauma (MST). $n=2261$ due to missing data for covariates

prospective risk of unintended pregnancy despite greater numbers of historical unintended pregnancies among women with mental health disorders. Indeed, VA may be particularly well-suited to provide contraceptive care to women with mental illness, due to the high prevalence of mental illness among the population it serves and innovations such as integration of mental health services within gender-specific primary care settings. ${ }^{30,31}$ As a result of this co-localization, women seeking mental health care may actually have increased opportunities for contraceptive counseling and provision of prescription contraception. With regard to historical unintended pregnancies, Veterans may have had differential access to contraception prior to military service or VA enrollment, and prior reproductive experiences such as unintended pregnancy may also influence current contraceptive choices. ${ }^{32}$

This study contributes to the literature by examining the relationship between mental illness and the number of unintended pregnancies women have experienced, a topic which has received little attention to date. A systematic review of seven studies performed in the USA found that greater age, Black or Hispanic race, non-voluntary first intercourse, previous abortion, and history of trading sex for money or resources were associated with reporting multiple unintended pregnancies, ${ }^{33}$ however, none of these studies assessed mental illness as a potential predictor. In our sample, $29 \%$ of women had experienced more than one unintended pregnancy, in line with estimates in the general population, which range from 17 to $32 \% .{ }^{34,35}$ Women with any mental health disorder, and particularly those reporting greater than one condition, were more likely to have experienced higher numbers of unintended pregnancies than were women without mental illness, even after adjusting for numerous potential confounders. This suggests that mental illness may be a novel predictor of multiple unintended pregnancies, at least in a Veteran population. Whereas experiencing one unintended pregnancy may simply reflect probabilities of contraceptive failure, multiple

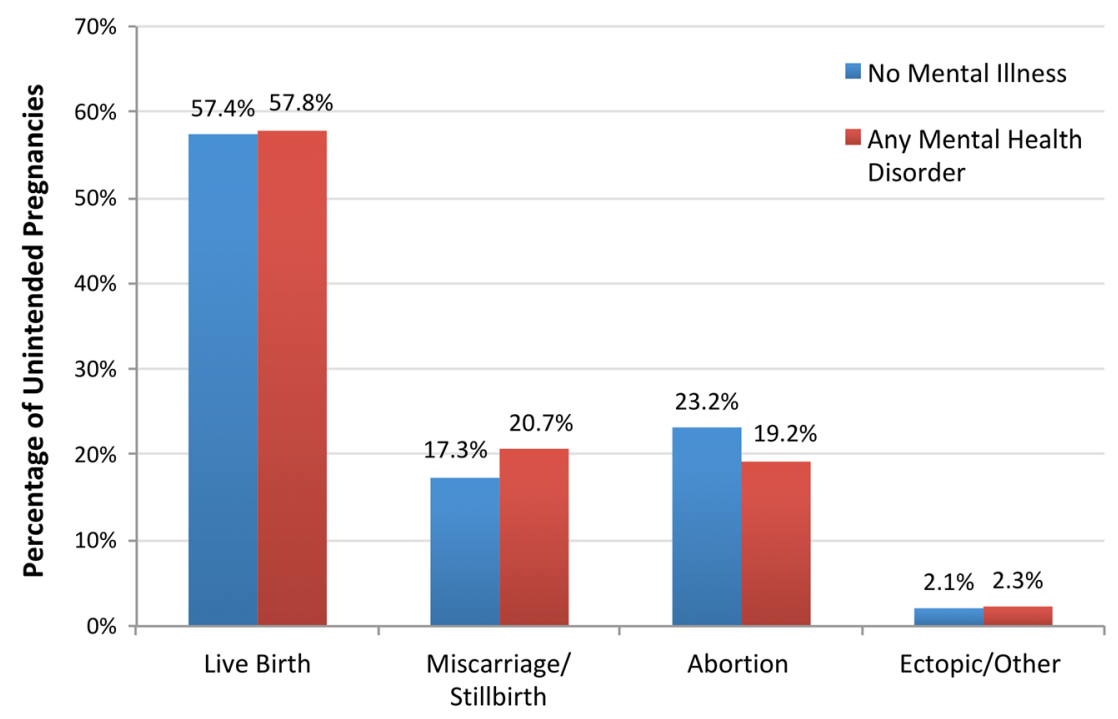

Fig. 2 Outcomes of completed unintended pregnancies by any mental health disorder. $n=2489$ unintended pregnancies reported by 1308 women. Overall $p=0.07$, adjusting for clustering of pregnancies by woman 
Table 4 Contraceptive use at last sex by any mental health disorder among heterosexually active women Veterans not desiring pregnancy $(n=$ 1339)

A) Bivariate analyses

No mental health

disorder $(n=455,34.0 \%)$

Any mental health

disorder $(n=884,66.0 \%)$

B) Adjusted models

Any contraceptive

use vs. none

aOR $(95 \% \mathrm{CI}) \|$

Any mental health disorder vs. none $0.74(0.42,1.29)$

Any contraceptive use

$n(\%)$

$435(95.6 \%)$

$829(93.8 \%)$

interval

aOR adjusted odds ratio CI confidence interval

*Highly effective methods: male or female sterilization, intrauterine device (IUD) or implant; moderately effective methods: pill, patch, ring, DepoProvera; least effective methods: barrier methods, fertility awareness, withdrawal

tp values are from chi-square tests

$\neq$ Logistic regression model for any contraceptive use vs. none by any mental health disorder; adjusted $p$ value $0.29 ; n=1317$ due to missing data for covariates

$\S$ Multinomial regression model for contraceptive efficacy by any mental health disorder; reference group = least effective methods; overall adjusted $p$ value $0.13 ; n=1244$ due to missing data for covariates

\|Adjusted for age, racelethnicity, marital status, education, annual household income, religion, additional (non-VA) insurance, census region, deployment history, and history of military sexual trauma (MST)

unintended pregnancies may reflect a different mechanism associated with this population, such as reduced contraceptive adherence or greater pregnancy ambivalence. More research is needed to clarify the potential role of mental illness in predicting higher-order unintended pregnancies.

The prevalence of overall and individual mental health disorders observed in this study is notably higher than existing estimates based on VA administrative data, ${ }^{1,16}$ likely due to our use of self-reported survey data and focus on lifetime incidence. While we were unable to perform multivariable analyses for individual mental health disorders, our bivariate results suggest that depression, either individually or in combination with other disorders, may be more strongly associated with unintended pregnancy than are other mental health conditions. This finding is consistent with research indicating that depressive symptoms in particular are predictive of inconsistent or nonuse of contraception and subsequent unintended pregnancy, ${ }^{12,13,17,18,36}$ and that unintended pregnancy is associated with perinatal depression. ${ }^{24}$

This study has several limitations. Reliance on selfreported mental health disorders may overestimate the prevalence of diagnosed conditions, while small sample sizes for women with a single diagnosis limits our ability to assess the specificity of associations between individual conditions and unintended pregnancy. Our cohort of female VA-users limits the generalizability of our results to the general US population, as well as to Veterans who do not use VA for health care. Although we used standard items to assess pregnancy intention, ${ }^{14}$ these measures may be subject to recall bias. Furthermore, despite our use of unintended pregnancy as an adverse outcome measure, unintended pregnancies may not be universally negative events for all women. Timing-based definitions of unintended pregnancy do not account for emotional orientations or responses to existing pregnancies, and a wide body of research suggests that some women experience an unintended pregnancy as positive or welcome. ${ }^{37,38}$ This possibility is particularly relevant given the cross-sectional nature of our data, which limits our ability to assess the temporal relationship between unintended pregnancy and onset of mental health disorders. We are also unable to assess co-occurring vs. sequentially experienced mental health disorders, which may influence the impact of mental illness burden on unintended pregnancy. Future work should include additional exploration of adherence to user-dependent methods among women Veterans, and prospective, longitudinal studies to examine the impact of specific mental health diagnoses and symptomology on contraceptive use and risk of unintended pregnancy. Ongoing work aimed at better understanding the reproductive goals, preferences, and unique needs of women Veterans with mental health disorders is also needed to inform clinical practices that best support these women.

In conclusion, women Veterans report high rates of both mental health disorders and unintended pregnancies, and the two are highly associated. Additional research is needed to further elucidate mechanisms underlying this relationship, in order to inform interventions to improve reproductive health outcomes and enable clinicians to better support women in reaching their reproductive goals. Such interventions will be particularly important for women Veterans and other populations with a high burden of mental illness. 
Financial Support: The ECUUN study was supported by the United States Department of Veterans Affairs, Health Services Research and Development Service (HSR\&D), Merit Review Award IIR 12-124 (PI: Sonya Borrero). Colleen Judge is supported by the National Center for Advancing Translational Sciences of the National Institutes of Health under Award Number TL1TR001858 (PI: Wishwa Kapoor). Lisa Callegari was supported by a Health Services Research and Development Career Development Award (CDA 14-412).

The findings and conclusions in this report are those of the authors and do not represent the views of the Department of Veterans Affairs, the United States Government, or the National Institutes of Health.

Prior Presentations: Data included in this manuscript were presented as a poster presentation at the North American Forum on Family Planning (the Forum), November 14-16, 2015, Chicago, IL.

Corresponding Author: Colleen P. Judge-Golden, BA; University of Pittsburgh School of Medicine, Pittsburgh, PA, USA (e-mail: cpj6@pitt. edu).

\section{Compliance with Ethical Standards:}

The institutional review boards of VA Pittsburgh Healthcare System and the University of Pittsburgh approved this study.

Conflict of Interest: The authors declare that they do not have a conflict of interest.

\section{REFERENCES}

1. Frayne S, Phibbs C, Saechao F, et al. Sourcebook: Women Veterans in the Veterans Health Administration. Volume 3. Sociodemographics, Utilization, Costs of Care, and Health Profile. Washington, DC: Women's Health Evaluation Initiative, Women's Health Services, Veterans Health Administration, Department of Veterans Affairs; 2014.

2. Friedman SA, Phibbs CS, Schmitt SK, Hayes PM, Herrera L, Frayne SM. New Women Veterans in the VHA: A Longitudinal Profile. Women Health Iss. 2011;21:S103-S11.

3. Borrero S, Callegari LS, Zhao $\mathbf{x}$, et al. Unintended Pregnancy and Contraceptive Use Among Women Veterans: The ECUUN Study. J Gen Intern Med. 2017;32:900-8.

4. Gipson JD, Koenig MA, Hindin MJ. The Effects of Unintended Pregnancy on Infant, Child, and Parental Health: A Review of the Literature. Stud Fam Plann. 2008;39:18-38.

5. Eisen SV, Schultz MR, Vogt D, et al. Mental and physical health status and alcohol and drug use following return from deployment to Iraq or Afghanistan. Am J Public Health. 2012;102 Suppl 1:S66-73.

6. Lehavot $\mathbf{K}$, Hoerster KD, Nelson KM, Jakupcak M, Simpson TL Health Indicators for Military, Veteran, and Civilian Women. Am J Prev Med. 2012;42:473-80.

7. Blosnich JR, Dichter ME, Cerulli C, Batten SV, Bossarte RM. Disparities in adverse childhood experiences among individuals with a history of military service. JAMA Psychiatry. 2014;71:1041-8.

8. Byrne T, Montgomery AE, Dichter ME. Homelessness among female veterans: a systematic review of the literature. Women Health. 2013;53:572-96.

9. Dunkel Schetter C, Tanner L. Anxiety, depression and stress in pregnancy: implications for mothers, children, research, and practice. Curr Opin Psychiatry. 2012;25:141-8.

10. Grote NK, Bridge JA, Gavin AR, Melville JL, Iyengar S, Katon WJ. A meta-analysis of depression during pregnancy and the risk of preterm birth, low birth weight, and intrauterine growth restriction. Arch Gen Psychiatry. 2010;67:1012-24.

11. Rogal SS, Poschman $\mathbf{K}$, Belanger $\mathbf{K}$, et al. Effects of posttraumatic stress disorder on pregnancy outcomes. J Affect Disord. 2007;102:137-43.

12. Hall KS, Kusunoki Y, Gatny H, Barber J. The risk of unintended pregnancy among young women with mental health symptoms. Soc Sci Med. 2014;100:62-71.

13. James-Hawkins L, Denardo D, Blalock C, Mollborn S. Do Depressive Symptoms in Male and Female Adolescents Predict Unintended Births in Emerging Adulthood? Matern Child Health J. 2014;18:2115-23.

14. 2013-2015 NSFG: Public Use Data Files, Codebooks, and Documentation. Available at: http://www.cdc.gov/nchs/nsfg/nsfg_2013_2015_puf. htm. Accessed Feb 1, 2018.
15. Berenson $\mathbf{A B}$, Breitkopf $\mathbf{C R}$, Wu ZH. Reproductive correlates of depressive symptoms among low-income minority women. Obstet Gynecol. 2003; 102:1310-7.

16. Callegari LS, Zhao X, Nelson KM, Borrero S. Contraceptive adherence among women Veterans with mental illness and substance use disorder. Contraception. 2015;91:386-92.

17. Hall KS, Moreau C, Trussell J, Barber J. Role of young women's depression and stress symptoms in their weekly use and nonuse of contraceptive methods. J Adolesc Health. 2013;53:241-8.

18. Hall KS, Moreau C, Trussell J, Barber J. Young women's consistency of contraceptive use - Does depression or stress matter? Contraception. 2013;88:641-9.

19. Hall KS, White KOC, Rickert VI, Reame N, Westhoff C. Influence of depressed mood and psychological stress symptoms on perceived oral contraceptive side effects and discontinuation in young minority women. Contraception. 2012;86:518-25.

20. Patel PR, Laz TH, Berenson AB Patient characteristics associated with pregnancy ambivalence. J Womens Health (Larchmt). 2015;24:37-41.

21. Francis $\mathbf{J}$, Malbon $\mathbf{K}$, Braun-Courville $\mathbf{D}$, Lourdes LO, Santelli $\mathbf{J}$. Ambivalence About Pregnancy and Its Association With Symptoms of Depression in Adolescent Females Initiating Contraception. J Adolesc Health. 2015;56:44-51.

22. Higgins JA, Popkin RA, Santelli JS. Pregnancy Ambivalence and Contraceptive Use Among Young Adults in the United States. Perspect Sex Reprod Health. 2012;44:236-43.

23. Schwarz EB, Lohr PA, Gold MA, Gerbert B. Prevalence and correlates of ambivalence towards pregnancy among nonpregnant women. Contraception. 2007;75:305-10.

24. Abajobir AA, Maravilla JC, Alati R, Najman JM. A systematic review and meta-analysis of the association between unintended pregnancy and perinatal depression. J Affect Disord. 2016;192:56-63.

25. Bahk J, Yun S-C, Kim Y-m, Khang Y-H. Impact of unintended pregnancy on maternal mental health: a causal analysis using follow up data of the Panel Study on Korean Children (PSKC). BMC Pregnancy Childbirth. 2015; 15:85.

26. Fellenzer JL, Cibula DA. Intendedness of pregnancy and other predictive factors for symptoms of prenatal depression in a population-based study. Matern Child Health J. 2014;18:2426-36.

27. Herd P, Higgins J, Sicinski K, Merkurieva I. The Implications of Unintended Pregnancies for Mental Health in Later Life. Am J Public Health. 2016;106:421-9.

28. Kazerooni R, Blake A, Thai J. Predictors of Pregnancy in Female Veterans Receiving a Hormonal Contraceptive Pill, Patch, or Ring. Ann Pharmacother. 2015;49:1284-90.

29. Callegari LS, Zhao X, Nelson KM, Lehavot K, Bradley KA, Borrero S. Associations of mental illness and substance use disorders with prescription contraception use among women veterans. Contraception. 2014;90:97-103.

30. Leung LB, Yoon J, Escarce JJ, et al. Primary Care-Mental Health Integration in the VA: Shifting Mental Health Services for Common Mental Illnesses to Primary Care. Psychiatric services (Washington, D.C.). 2018;69:403-9.

31. Wray LO, Szymanski BR, Kearney LK, McCarthy JF. Implementation of primary care-mental health integration services in the Veterans Health Administration: program activity and associations with engagement in specialty mental health services. Journal of clinical psychology in medical settings. 2012;19:105-16.

32. Dehlendorf C, Park SY, Emeremni CA, Comer D, Vincett $K$, Borrero S. Racial/ethnic disparities in contraceptive use: variation by age and women's reproductive experiences. Am J Obstet Gynecol. 2014;210:526 e1-9.

33. Aztlan-James EA, McLemore M, Taylor D. Multiple Unintended Pregnancies in U.S. Women: A Systematic Review. Women Health Iss. 2017;27:407-13.

34. Jones RK, Singh S, Finer LB, Frohwirth LF. Repeat Abortion in the United States. New York, NY: Guttmacher Institute;2006.

35. Magnusson BM, Masho SW, Lapane KL. Adolescent and sexual history factors influencing reproductive control among women aged 18-44. Sex Health. 2011;8:95-101.

36. Aztlan EA, Foster DG, Upadhyay U. Subsequent Unintended Pregnancy Among US Women Who Receive or Are Denied a Wanted Abortion. Journal of midwifery \& women's health. 2018;63:45-52.

37. Aiken AR, Dillaway C, Mevs-Korff $\mathbf{N}$. A blessing I can't afford: factors underlying the paradox of happiness about unintended pregnancy. Soc Sci Med. 2015; 132:149-55.

38. Borrero S, Nikolajski C, Steinberg $\mathbf{J R}$, et al. "It just happens": a qualitative study exploring low-income women's perspectives on pregnancy intention and planning. Contraception. 2015;91:150-6. 